\title{
Advances in the development of a Cherenkov diagnostic system to study runaway electron losses
}

\author{
Federica Causa* \\ Unità Tecnica Fusione, ENEA C. R. Frascati, via E. Fermi 45, 00044 Frascati (Roma), Italy \\ E-mail: federica.causalenea.it
}

\section{Gianluca Pucella}

Unità Tecnica Fusione, ENEA C. R. Frascati, via E. Fermi 45, 00044 Frascati (Roma), Italy

E-mail: gianluca.pucella@enea.it

\section{Basilio Esposito}

Unità Tecnica Fusione, ENEA C. R. Frascati, via E. Fermi 45, 00044 Frascati (Roma), Italy E-mail: basilio.esposito@enea.it

\section{Paolo Buratti}

Unità Tecnica Fusione, ENEA C. R. Frascati, via E. Fermi 45, 00044 Frascati (Roma), Italy E-mail: paolo.buratti@enea.it

\section{Edmondo Giovannozzi}

Unità Tecnica Fusione, ENEA C. R. Frascati, via E. Fermi 45, 00044 Frascati (Roma), Italy E-mail: edmondo.giovannozzi@enea.it

\section{and the FTU Team}

See the appendix of G. Pucella et al., Proceedings of the 25th IAEA Fusion Energy Conf., St Petersburg, Russia, 13-18 $8^{\text {th }}$ October 2014

\section{Lech Jakubowski}

National Centre for Nuclear Research (NCBJ), 7 Andrzeja Soltana Str., 05-400 Otwock, Poland E-mail: l.jakubowski@ncbj.gov.pl

\section{Karol Malinowski}

National Centre for Nuclear Research (NCBJ), 7 Andrzeja Soltana Str., 05-400 Otwock, Poland E-mail: k.malinowski@ncbj.gov.pl

\section{Marek Rabinski}

National Centre for Nuclear Research (NCBJ), 7 Andrzeja Soltana Str., 05-400 Otwock, Poland E-mail: m.Rabinski@ncbj.gov.pl

\section{Marek J. Sadowski}

National Centre for Nuclear Research (NCBJ), 7 Andrzeja Soltana Str., 05-400 Otwock, Poland E-mail: m.j.sadowski@ncbj.gov.pl

\section{Jaroslaw Zebrowski}

National Centre for Nuclear Research (NCBJ), 7 Andrzeja Soltana Str., 05-400 Otwock, Poland E-mail: j.zebrowski@ncbj.gov.pl 
Uncontrolled rapid loss of runaway current represents one of the most critical issues in tokamak reactors. Significant research efforts are necessary to study and understand efficient ways to control Runaway Electrons (REs) and to implement adequate control schemes to prevent disruptions. In this context, the aim of the study presented here is to identify the potential of a Cherenkov optical diagnostics in detecting runaway electron losses in the presence of magnetic islands, in a scenario that is more controllable than a disruption. The Cherenkov probe was recently installed and tested in FTU in collaboration with the Polish National Centre for Nuclear Research (NCBJ). Data interpretation and correlation with several other diagnostics operating in FTU, brought out the high level of detail provided by the Cerenkov probe in detecting RE losses driven by magnetic islands. The development of the diagnostic system was designed to enable RE energy-discrimination and simultaneous detection at two toroidal/poloidal positions to evaluate the potential of this diagnostic system for implementation in next-generation fusion reactors. The demonstration and testing of the improved diagnostic system is planned for the upcoming FTU experimental campaign.

First EPs Conference on Plasma Diagnostics - 1st ECPD,

14-17 April 2015

Villa Mondragone, Frascati (Rome) Italy

${ }^{*}$ Speaker. 


\section{Introduction}

Post-disruption events represent a major concern for the safety of large tokamaks because uncontrolled rapid loss of runaway current may irreparably damage plasma-facing components, e.g., by ablating high- $\mathrm{Z}$ materials such as Tungsten, and by reaching cooling ducts behind low- $\mathrm{Z}$ materials such as Beryllium. Significant research efforts are necessary to study and understand how to control Runaway Electrons (REs) and to implement adequate control schemes to prevent disruptions. Fast electrons within the plasma are typically monitored and detected using infrared, [1], gamma-ray cameras and hard X-ray diagnostics, [2], [3]. However, recently an optical diagnostics based on the Cherenkov effect, [4], was demonstrated to be effective in monitoring fast electron losses in FTU, particularly in scenarios involving plasma instabilities that can lead to disruption, [5]. The installation and test of the Cerenkov probe in FTU was done in collaboration with the Polish National Centre for Nuclear Research (NCBJ), [6]. Data interpretation and correlation with several other diagnostics operating in FTU, brought out the high level of detail provided by the Cerenkov probe in detecting RE losses driven by magnetic islands, [3]. In this type of scenario, the Cherenkov signal not only is modulated in synchronism with island rotation, but also reveals an internal structure of the signal peaks. This level of detail, achieved by the fast acquisition afforded by such an optical diagnostic technique, can provide further insight into the mechanism of RE loss in this scenario and, importantly, the identification of precursors leading to disruptions, thus leading to more effective real-time control measures.
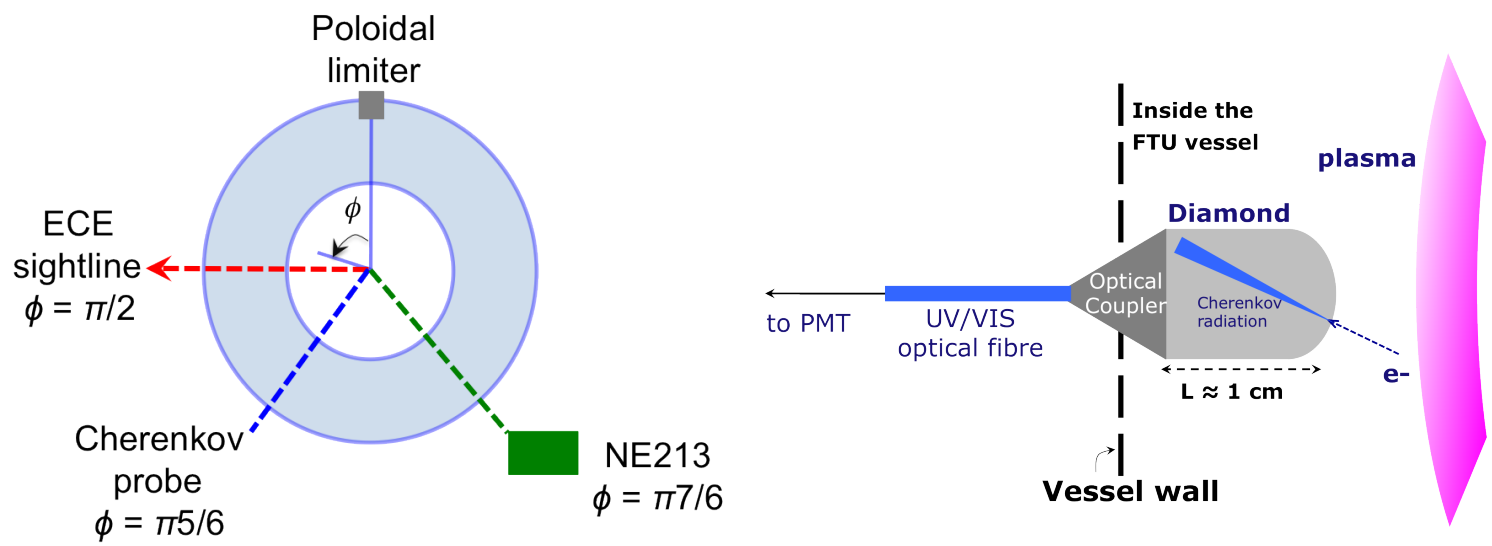

Figure 1: Top view, simplified schematics of (left) FTU equatorial section with positions of relevant diagnostics and (right) Cherenkov detector (not to scale) and its position (L) with respect to the vessel wall.

\section{Cherenkov probe and other relevant diagnostics}

The Cherenkov probe consists of a diamond detector mounted on a TZM (99\% Mo, $0.5 \% \mathrm{Ti}, 0.1 \%$ $\mathrm{Zr}$ ) head inserted in the FTU vessel. The synthetic, single crystal diamond (10 mm diameter, $1 \mathrm{~mm}$ thickness) exposed to the plasma is coated with a 100/200/1000 nm Ti/Pt/Au interlayer which has the dual function of permitting possible future depositions of Mo layers to increase the electron energy threshold, and to filter out visible light, particularly the plasma $D_{\alpha}$ line. The latter property 
was verified experimentally by the total lack of signal from the Cherenkov probe in the presence of a plasma with strong visible emission, but no or low levels of runaway electrons. The detector head was positioned inside FTU with the long side of the slit (approximately $0.1 \mathrm{~cm} \times 1.2 \mathrm{~cm}$ ) parallel to the equatorial plane of the tokamak, Figure 1. Electrons impinging on the probe emit Cherenkov radiation in the diamond if they are superluminal, that is, if their speed is higher than the speed of light in the medium, a condition equivalent, in the present system, to a $58 \mathrm{keV}$ electron energy threshold. The Cherenkov radiation emitted within the diamond was coupled to a VIS/UV optical fibre connected to a high-gain photomultiplier tube operating in the $160-650 \mathrm{~nm}$ spectral range and characterised by a $2 \mathrm{~ns}$ response time. FTU is a compact, high field (up to $\mathrm{B}=8 \mathrm{~T}$ ) tokamak, with major radius $R_{0}=0.935 \mathrm{~m}$ and minor radius a $=0.3 \mathrm{~m}$. The diamond probe was inserted inside the vessel at a variable radial position in the limiter shadow, on the equatorial plane of FTU, at $\phi=$ $150 \mathrm{deg}$, Figure 1. The data acquisition rate was $2 \mathrm{MHz}$ over the duration of the discharge (up to $2 \mathrm{~s}$ ), thus resulting in a high temporal resolution comparable to or better than that of other relevant diagnostics available in FTU. In FTU, a liquid organic proton recoil scintillator (NE213) and a proportional counter (BF3) are used to monitor, respectively, gamma and neutron emissions, [3]. The NE213 scintillator is located outside the cryostat on the equatorial plane, at $\phi=210 \mathrm{deg}$, and is sensitive to both neutron and gamma-rays. Six BF3 proportional counters are used, positioned above the tokamak, insensitive to gamma-rays. NE213 and BF3 have been cross-calibrated such that their outputs are identical in the absence of gamma rays. Since gamma-ray contamination is significant only in discharges with REs, the gamma ray contribution due to the presence of REs can be obtained by subtracting the neutron baseline (BF3) from the neutron and gamma integrated signal (NE213). In addition, since the maximum neutron rate possible with BF3 is $10^{12}$ neutron/s, three U-235 fission chambers located on the equatorial plane, at 120 deg separation, are used to extend the neutron dynamic range and monitor high neutron rates up to $10^{16}$ neutron/s, with $1 \mathrm{~ms}$ resolution. The plasma magnetic activity is monitored by means of a set of poloidal field pick-up (Mirnov) coils. In particular, the poloidal $(\mathrm{m})$ and toroidal $(\mathrm{n})$ periodicity numbers of helical magnetic islands chains generated by tearing instabilities are determined by cross-phase measurements. Further information on the internal island structure is given by temperature profile oscillations, as measured by a twelve-channel Electron Cyclotron Emission (ECE) diagnostic and by a soft x-ray pinhole camera. The ECE sightline is located on the equatorial plane, at 90 deg toroidal distance from the poloidal limiter and $60 \mathrm{deg}$ distance from the Cherenkov probe, having defined increasing angles in the counter clokwise direction as seen from above the equatorial plane, Figure 1.

\section{Scenario analysis}

Data from the Cherenkov probe on FTU was analysed in a range of scenarios. In all cases the Cherenkov signals could be clearly correlated with those from other diagnostics, but importantly, the signals display characteristic features related to the physical mechanisms underlying the different scenarios (continuous, bursty or island-modulated RE transport). However, the interest here is in scenarios with tearing modes developing magnetic islands where enhanced RE losses can be observed, e.g., [7], [8], and correlated with the passage of the island O-point in front of the probe, to study the dynamics of non-thermal electrons in the presence of magnetic islands and identify key mechanisms of RE generation due to magnetic perturbations. In the presence of MHD activity 

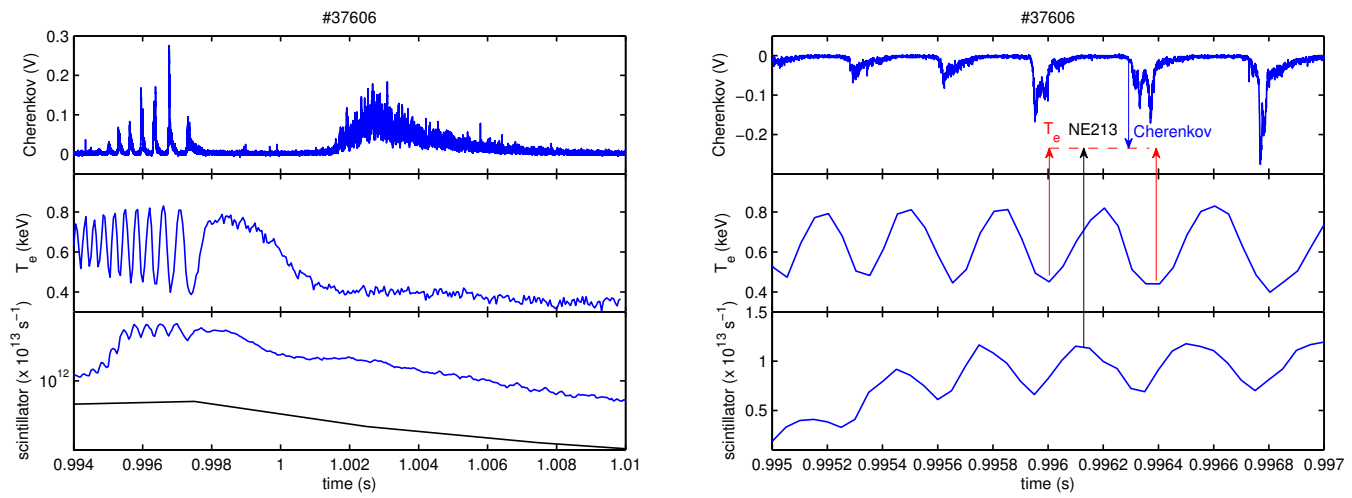

Figure 2: (Left) Scenario involving tearing modes growth and locking of a magnetic island and the modulation of Cherenkov, ECE and gamma-ray signals during island rotation in FTU pulse 37606 (B = 5.3 T, I = 520 kA): Cherenkov signal (top panel), electron temperature (ECE channel 11) (middle panel), NE213 (lower panel) and BF3 (lower panel, black line) signals and (Right) Relative timing of the signals; the Cherenkov signal is inverted and the BF3 neutron base line, with signal level $10^{11} s^{-1}$, is not shown to emphasise the modulation of NE213.

consisting in the growth and locking of a magnetic island with poloidal and toroidal periodicity numbers $(m, n)=(2,1)$, a clear phase-relation was identified between Cherenkov, ECE and NE213 scintillator signals, showing that the modulation of the Cherenkov signal is due to the rotation of the magnetic island, Figure 2. From the phase lags it is observed that the Cherenkov probe detects a signal peak only when the island O-point is passing in the proximity of the probe. In fact there is a 60 deg phase delay between the O-point passage at the ECE sightline and the Cherenkov signal peak, corresponding to the toroidal distance between the two diagnostics. This observation is further substantiated by the nearly antiphase correlation observed between the Cherenkov probe and NE213: the latter is sensitive to the total radiation (no spatial resolution) emitted by escaping REs, so its signal is maximum when the island is interacting with the poloidal limiter which is almost diametrically opposite to the Cherenkov probe. The last rotation of the island before disruption is clearly seen by the Cherenkov probe as demonstrated by the large signal in the time interval $1.002-1.006 \mathrm{~s}$, and also by the neutron diagnostics (NE213 and BF3). On the other hand, this event is not detected in the ECE signal because the associated temperature gradients are below the resolution of the diagnostics. Another aspect of interest is the internal structure of the peaks of the Cherenkov signal, with sub-peak full-width at half maximum of the order of 10-20 $\mu$ s: this level of detail opens up new possibilities for the investigation of fast electrons dynamics in the presence of high-amplitude magnetic islands. The main limitation of the present single-channel Cherenkov diagnostic system is that it provides an integral signal from one location within the vessel, providing no information on the spatial and energy distribution of incoming electrons. The planned upgrade of the diagnostic system is oriented to enable RE energy-discrimination and simultaneous detection at two toroidal/poloidal positions to evaluate the potential of this diagnostic system for implementation in next-generation fusion reactors. Hardware components have been ordered. Assembly of the head will be done at NCBJ in May 2015. The head will be shipped to ENEA, where it will be mounted on a suitably designed arm to permit rotation and translation of the head to control the 
orientation of the diamond window with respect to the equatorial plane of the tokamak, and the depth at which REs will be collected. Energy ranges of the three channels will be chosen to permit a meaningful correlation with existing diagnostics, such as the Fast Electron Bremsstrahlung camera. The probe is expected to be ready for laboratory and vacuum testing in October 2015. Subsequently it will be installed in FTU and tested in the upcoming experimental campaign.

\section{Conclusions}

A Cherenkov probe was successfully installed and tested on FTU. Signals from the Cherenkov detector were found to be correlated with those from other FTU diagnostics including Mirnov coils, ECE, neutron and gamma detectors. These results provide evidence of loss of confinement of electrons during magnetic reconnection, with a level of detail providing a deeper insight into the dynamics of the reconnection event. The Cherenkov probe upgrade that will enable energy and spatially resolved analysis of RE losses in FTU is planned for the next experimental campaign.

\section{Acknowledgement}

This work has been carried out within the framework of the EUROfusion Consortium and has received funding from the Euratom research and training programme 2014-2018 under grant agreement No 633053. The views and opinions expressed herein do not necessarily reflect those of the European Commission.

\section{References}

[1] R. Jaspers, N. J. Lopes Cardozo, A. J. H. Donné, H. L. M. Widdershoven and K. H. Finken, Rev. Sci. Instrum. 72, 466 (2001).

[2] O. Tudisco at al., The Diagnostic Systems in the FTU, Fusion Science and Technology, Chapter 8, 45(3), (2004), 402-421.

[3] B. Esposito et al, Dynamics of high energy runaway electrons in the Frascati Tokamak Upgrade, Phys. Plasmas 10 (2003) 2350.

[4] M. Rabinski et al., Feasibility study and design of Cherenkov-type detectors for measurements of fast electrons within tokamaks, Prob. At. Sci. Tech. 13 (2007) 206-208.

[5] P. Helander et al., Runaway acceleration during magnetic reconnection in tokamaks, Plasma Phys. Control. Fusion 44 (2002) B247-B262.

[6] L. Jakubowski et al., Cherenkov-type diamond detectors for measurements of fast electrons in the TORE-SUPRA tokamak, Rev. Sci. Inst. 81 (2010) 013504.

[7] R. J. Zhou et al., Effect of magnetic fluctuations on the confinement and dynamics of runaway electrons in the HT-7 tokamak, Physics of Plasmas 20 (2013) 032511

[8] Ivanov et al., Interdependence of Magnetic Islands, Halo Current and Runaways Electrons in T-10 Tokamak, IAEA-CN-77/EXP2/02 Sorrento, 2000 